\title{
A new approach to estimating weight change and its reference intervals during the first 96 hours of life
}

\author{
MJ Fonseca (mjfonseca@med.up.pt) ${ }^{1,2}$, M Severo ${ }^{1,2}$, AC Santos ${ }^{1,2}$ \\ 1.EPIUnit - Institute of Public Health, University of Porto, Porto, Portugal \\ 2.Department of Clinical Epidemiology, Predictive Medicine and Public Health, University of Porto Medical School, Porto, Portugal
}

\section{Keywords}

neonate, newborn, reference intervals, weight loss

\section{Correspondence}

M J Fonseca, Institute of Public Health - University of Porto

Rua das Taipas $n^{\circ} 135,4050-600$ Porto, Portugal.

Tel: (351) 222061820

Fax: (351) 222061821

Email: mjfonseca@med.up.pt

\section{Received}

30 July 2014; accepted 5 December 2014.

DOI:10.1111/apa.12894

\begin{abstract}
Aim: We aimed to apply a novel model to estimate weight change and its reference intervals during the first $96 \mathrm{~h}$ of life and the time of weight nadir.

Methods: This study involved 1288 full-term singletons, from the Generation XXI birth cohort. Recruitment occurred between 2005 and 2006 in all five public units providing obstetrical and neonatal care in Porto, Portugal. Birthweight was abstracted from clinical records, and the subsequent newborn anthropometrics were obtained by trained examiners. Longitudinal models to estimate postnatal weight were tested and the weight ratio was calculated as the weight during $96 \mathrm{~h}$ of life divided by birthweight.

Results: The chosen model was (weight $(t) \sim 3241.442+(-9.378) \times t+0.119 \times t^{2}+$ $0.000 \times t^{3}+b_{0 i}+b_{1 i} \times t$, where $t$ represented the newborn infant's age in hours and $b_{i}$ represented the random coefficients. The curve inflection point (nadir) was achieved at $52.3 \mathrm{~h}$ of life, corresponding to a loss of $218 \mathrm{~g}$ and a weight ratio of 0.933 . We estimated that at six, 12, 24 and $36 \mathrm{~h}$ of life the mean weight ratio and 10th-90th percentiles were 0.978 (0.968-0.988), 0.968 (0.953-0.983), 0.951 (0.928-0.974) and 0.939 (0.9090.969), respectively.
\end{abstract}

Conclusion: This model allows a more accurate estimate of newborn weight change and its reference intervals, and estimated the nadir at $52.3 \mathrm{~h}$ of life, corresponding to a weight ratio of 0.933 .

\section{INTRODUCTION}

During the first days of life, newborn infants lose around 6\% of their birthweight, and the second and third days seem to be those when the maximum weight loss takes place and newborn infants achieve their minimum weight (nadir) (1). This physiological weight loss is expected and mainly represents redistribution of fluids as a consequence of cardiopulmonary adaptation $(2,3)$. However, it may also involve fat loss due to the catabolic state of some newborn infants, such as those small for gestational age or when there is some delay in establishing breastfeeding.

Excessive weight loss has been associated with hypernatraemic dehydration, which can cause serious medical complications, such as disseminated intravascular coagulation, stroke and even death (4-7). On the other hand, insufficient weight loss, usually defined as weight gain or maintenance, has also been associated with health risks in the neonatal period, namely overhydration and related morbidities such as bronchopulmonary dysplasia, intraventricular-periventricular haemorrhage, necrotising enterocolitis and patent

Abbreviation

SD, standard deviation. ductus arteriosus (8-11). Despite these health risks, few studies have focused on this issue. In 2008, a systematic review (1) aimed to establish the reference weight loss during the first 2 weeks following birth. The authors only found 11 studies that evaluated the weight change in healthy, full-term and exclusively breastfed singletons and realised that the methods used to report it were inconsistent and that further research was needed (1). Most studies performed weight measurements on the day of birth and on specific days, for example on day three, while others measured their newborn infants daily. In both study methods, the nadir may not have

\section{Key notes}

- This is the first description of weight change during the first $96 \mathrm{~h}$ of life estimated by a mixed-effect model along with reference intervals.

- Newborn infants continuously lost weight until $52 \mathrm{~h}$ of life, corresponding to a $218 \mathrm{~g}$ weight loss and a 0.93 weight ratio, calculated as weight divided by birthweight.

- Using the estimated weight change curves, clinicians can rapidly observe whether a newborn infant is experiencing excessive weight loss and initiate primary interventions. 
been correctly assessed, because daily measurements may not be close enough.

Thus, we aimed to apply a novel model to estimate the weight change that occurs during the first $96 \mathrm{~h}$ of life, along with its reference intervals and the time in hours when the nadir is achieved, in a sample of full-term newborn infants.

\section{PATIENTS AND METHODS}

This study involved 1948 newborn infants recruited between November 2005 and August 2006 from the Generation XXI birth cohort (12). Only newborn infants weighted after birth were included. Briefly, participants were selected at the five public tertiary care maternity units providing obstetrical and neonatal care in the metropolitan area of Porto, Portugal.

Of the 1948 newborn infants, we excluded 39 from multiple births, 85 preterm infants with a gestational age of $<37$ weeks and 18 newborn infants with congenital anomalies. Of the remaining 1806 newborninfants, 465 had missinginformation on the time of measurement and six had incongruent values. We excluded 28 newborn infants measured after $96 \mathrm{~h}$ of life and 19 outliers, defined as the first/third quartile \pm three times the interquartile range. The final sample comprised 1288 newborn infants, whose characteristics are presented in Table 1. Differences regarding maternal, pregnancy, delivery and newborn infant's characteristics between the included $(n=1288)$ and excluded participants $(n=518)$ were tested and the only relevant difference was regarding the proportion of Caesarean section, which was higher among those excluded than included (35.0\% versus $29.2 \%, \mathrm{p}=0.017)$.

Data on newborn infant's characteristics, including birthweight, were abstracted from medical records by trained interviewers. Then, during the hospital stay, trained examiners weighed the newborn infants to the nearest $1 \mathrm{~g}$ and registered the date and time of measurement. There were $215(16.7 \%)$ newborn infants measured before $24 \mathrm{~h}$ of life, $566(43.9 \%)$ measured between 24 and $48 \mathrm{~h}$ of life, 388 $(30.1 \%)$ measured between 48 and $72 \mathrm{~h}$ of life, and 119 $(9.2 \%)$ measured after the $72 \mathrm{~h}$ of life. The time of measurement varied between 6.3 and $96 \mathrm{~h}$ of life, and the sample mean was $45.3 \mathrm{~h}$ of life (SD 19.4).

The study protocol was approved by the ethics committee of Hospital de S João and by the National Commission for Data Protection. Written informed consent was obtained from all mothers at recruitment.

\section{Statistical analysis}

Longitudinal models were tested to ascertain the best approach to calculate newborn weight change, because weight was highly dependent on the newborn infant's age when the measurement was performed. So, to estimate newborn weight independently of age, we tested mixed-effect models adjusted for the time of measurement in hours $(t)$, combining fixed coefficients up to the third degree and incorporating random effects on the intercept and slope (Table 2). We compared four longitudinal models: linear (weight $\left.(t) \sim \beta_{0}+\beta_{1} \times t+b_{0 i}\right)$, quadratic (weight $(t) \sim \beta_{0}+\beta_{1} \times t$
Table 1 Maternal, pregnancy, delivery and newborn infant's characteristics of the study sample

n $(\%)$

\begin{tabular}{|c|c|}
\hline \multicolumn{2}{|l|}{ Maternal characteristics } \\
\hline \multicolumn{2}{|l|}{ Age at delivery (years) } \\
\hline $13-17$ & $21(1.6)$ \\
\hline $18-29$ & $657(51.1)$ \\
\hline $30-34$ & $408(31.8)$ \\
\hline $35-39$ & $169(13.2)$ \\
\hline$\geq 40$ & $30(2.3)$ \\
\hline \multicolumn{2}{|l|}{ Prepregnancy BMI $\left(\mathrm{kg} / \mathrm{m}^{2}\right)$} \\
\hline$<18.50$ & $39(3.2)$ \\
\hline $18.50-24.99$ & $769(63.3)$ \\
\hline 25.00-29.99 & $303(25.0)$ \\
\hline$\geq 30.00$ & $103(8.5)$ \\
\hline Primipara & $725(56.3)$ \\
\hline \multicolumn{2}{|l|}{ Pregnancy characteristics } \\
\hline Tobacco smoke during third trimester & $179(14.2)$ \\
\hline Gestational diabetes & $44(3.5)$ \\
\hline Hypertension during pregnancy & $17(1.3)$ \\
\hline Weight gain (kg) [mean (SD)] & $13.5(5.61)$ \\
\hline \multicolumn{2}{|l|}{ Delivery characteristics } \\
\hline \multicolumn{2}{|l|}{ Type } \\
\hline Vaginal & $906(70.8)$ \\
\hline Caesarean & $374(29.2)$ \\
\hline \multicolumn{2}{|l|}{ Newborn infant's characteristics } \\
\hline \multicolumn{2}{|l|}{ Gestational age (weeks) } \\
\hline $37-38$ & $397(30.8)$ \\
\hline $39-40$ & $778(60.4)$ \\
\hline $41-43$ & $113(8.8)$ \\
\hline \multicolumn{2}{|l|}{ Sex } \\
\hline Male & $655(50.9)$ \\
\hline Female & $633(49.1)$ \\
\hline \multicolumn{2}{|l|}{ Birthweight (g) } \\
\hline$<2500$ & $49(3.8)$ \\
\hline $2500-4000$ & $1192(92.5)$ \\
\hline$>4000$ & $47(3.6)$ \\
\hline Apgar score 1st minute $<7$ & $47(3.7)$ \\
\hline
\end{tabular}

[Correction added on 30 July 2015, after online publication: The values in " $n$ (\%)" column for age groups 13-17 and 18-19 were changed to "2 1 (1.6)" and "657 (51.1)", respectively.]

$\left.+\beta_{2} \times t^{2}+b_{0 i}\right)$, and cubic (weight $(t) \sim \beta_{0}+\beta_{1} \times t+\beta_{2} \times t^{2}+\beta_{3}$ $\times t^{3}+b_{0 i}$ ) with only a random intercept and the same cubic model but with random intercept and slope (weight $(t) \sim \beta_{0}+$ $\left.\beta_{1} \times t+\beta_{2} \times t^{2}+\beta_{3} \times t^{3}+b_{0 i}+b_{1 i} \times t\right)$. Weight change was then expressed as weight difference in grams (birthweight minus weight) and as weight ratio (weight divided by birthweight). As mixed-effect models were used to estimate weight, the methodology proposed by Royston (13) was applied to estimate age-related reference intervals for weight and weight difference. Considering that the variability of weight ratio with age showed a nonlinear relation, the methodology proposed by Altman (14) was used to estimate the age-related reference intervals using absolute residuals. Also, 95\% confidence intervals were estimated for weight ratio.

To facilitate the comparison with previous results, weight change percentage was also calculated as (weight minus birthweight) divided by birthweight. 
Table 2 Longitudinal models to ascertain the best approach to calculate newborn infant's weight at $t$ hours of life

\begin{tabular}{|c|c|c|c|c|}
\hline & & $\mathrm{p}$ & AIC & $\mathrm{BIC}$ \\
\hline Model 1: & weight $(t) \sim \beta_{0}+\beta_{1} \times t+b_{0 i}$ & - & 35400.80 & 35424.25 \\
\hline Model 2: & weight $(t) \sim \beta_{0}+\beta_{1} \times t+\beta_{2} \times t^{2}+b_{0 i}$ & $<0.001$ & 34890.89 & 34920.21 \\
\hline Model 3: & weight $(t) \sim \beta_{0}+\beta_{1} \times t+\beta_{2} \times t^{2}+\beta_{3} \times t^{3}+\mathrm{b}_{0 \mathrm{i}}$ & $<0.001$ & 34881.34 & 34916.52 \\
\hline Model 4: & weight $(t) \sim \beta_{0}+\beta_{1} \times t+\beta_{2} \times t^{2}+\beta_{3} \times t^{3}+\mathrm{b}_{0 \mathrm{i}}+\mathrm{b}_{1 \mathrm{i}} \times t$ & $<0.001$ & 34664.01 & 34710.92 \\
\hline
\end{tabular}

$\mathrm{AIC}=$ Akaike information criterion; $\mathrm{BIC}=$ Bayesian information criterion; $\beta_{\mathrm{i}}=$ fixed coefficients; $\mathrm{b}_{\mathrm{i}}=$ random coefficients; $t=$ time in hours.

Analyses were performed using IBM SPSS Statistics (version 21.0, SPSS Inc., Chicago, IL, USA) and R (version 2.14.1, R Foundation for Statistical Computing, Vienna, Austria).

\section{RESULTS}

The final model to estimate weight change during the first $96 \mathrm{~h}$ of life in full-term singletons was (weight $(\mathrm{t}) \sim 3241.442+$ $(-9.378) \times t+0.119 \times t^{2}+0.000 \times t^{3}+b_{0 i}+b_{1 i} \times t$, where $t$ represents the newborn infant's age in hours, $b_{0 i}$ the random intercept and $b_{1 i}$ the random slope. This was the chosen model because it had the lowest Akaike information criterion and Bayesian information criterion (Table 2).

To calculate the time when the weight was minimum (nadir), we used the following equation:

$$
\begin{aligned}
(\operatorname{weight}(t))^{\prime}= & 0(=) t \\
& =\frac{-2 \times \beta_{2} \pm \sqrt{\left(2 \times \beta_{2}\right)^{2}-4 \times\left(3 \times \beta_{3}\right) \times \beta_{1}}}{2 \times\left(3 \times \beta_{3}\right)} \\
& (=) t=52.3 \text { hours. }
\end{aligned}
$$

To estimate the minimum weight for each child, we replaced the $t$ in the equation by 52.3 , because it was the estimated nadir time. Figure 1 represents the curves for newborn infant's mean weight change expressed as weight ratio and its 95\% confidence interval and percentiles three, 10,90 and 97 estimated by the model. The maximum weight loss corresponds to the inflection point of the curve and was estimated as a weight ratio of 0.933 , occurring at $52.3 \mathrm{~h}$ of life. At this time point, the third percentile corresponded to a weight ratio of 0.878 , the 10 th percentile to 0.896 , the 90th percentile to 0.970 and the 97 th percentile to 0.987 .

Table 3 shows the estimated mean of newborn infant's weight and weight change, expressed as weight difference in grams and weight ratio, according to the newborn infant's age in hours and reference intervals. The estimated mean birthweight was $3241 \mathrm{~g}$ and newborn infants began to lose weight immediately after birth. At $6 \mathrm{~h}$ of life, the weight ratio was already 0.978 , at $24 \mathrm{~h} 0.951$ and at $36 \mathrm{~h} 0.939$. At $52.3 \mathrm{~h}$ of life, newborn infants achieved their minimum weight, corresponding to a mean loss of $218 \mathrm{~g}$ and a weight ratio of 0.933 and then they began to regain weight.

The distribution of the newborn infants according to their maximum weight change percentage is depicted in Table 4 . A weight loss equal or higher than $10 \%$ of birthweight

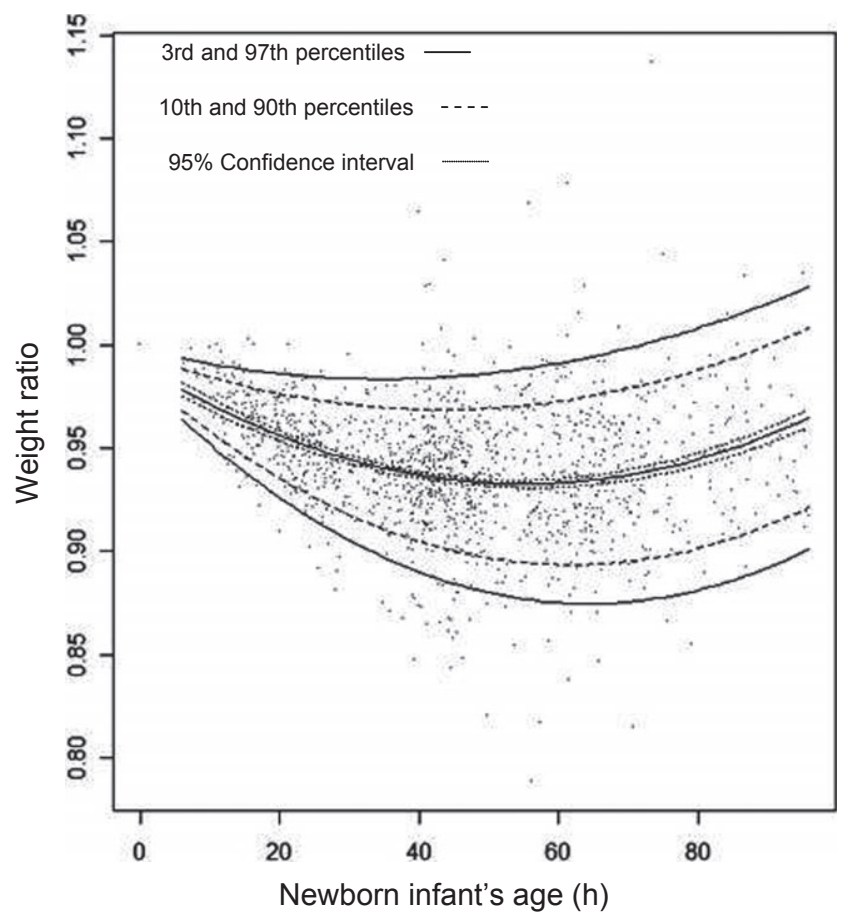

Figure 1 Curves for newborn infant's mean weight change expressed as weight ratio and its 95\% confidence interval and percentiles 3, 10,90 and 97 estimated by the model.

occurred in 6.1\% of the newborn infants: $5.7 \%$ lost between $10 \%$ and $15 \%$ of their birthweight and $0.4 \%$ of the newborn infants lost $15 \%$ or more of their birthweight. On the other hand, $0.6 \%$ of the newborn infants did not lose any weight, starting to gain weight immediately after birth.

\section{DISCUSSION}

In this sample of full-term Portuguese singletons, the mean nadir corresponded to a mean loss of $218 \mathrm{~g}$ and a weight ratio of 0.933 , occurring at $52.3 \mathrm{~h}$ of life, which is in accordance with previous studies (1). In our sample, newborn infants began to lose weight immediately after birth, being the weight ratio already of 0.978 at $6 \mathrm{~h}$ of life. After the $52.3 \mathrm{~h}$ of life, newborn infants started to gain weight.

A previous systematic review (1) that aimed to establish newborn weight change was performed in 2008 , and the 


\begin{tabular}{|c|c|c|c|c|c|c|c|c|c|c|c|c|c|c|c|}
\hline \multirow{3}{*}{$\begin{array}{l}\text { Newborn infant's } \\
\text { age } t \text { (hours) }\end{array}$} & \multicolumn{5}{|c|}{ Weight at $t$ hours (g) } & \multicolumn{5}{|c|}{ Weight difference at $t$ hours (g) } & \multicolumn{5}{|c|}{ Weight ratio at $t$ hours } \\
\hline & \multirow[b]{2}{*}{ Mean } & \multicolumn{4}{|c|}{ Percentiles } & \multirow[b]{2}{*}{ Mean } & \multicolumn{4}{|c|}{ Percentiles } & \multirow[b]{2}{*}{ Mean } & \multicolumn{4}{|c|}{ Percentiles } \\
\hline & & 3 & 10 & 90 & 97 & & 3 & 10 & 90 & 97 & & 3 & 10 & 90 & 97 \\
\hline 0 & 3241 & - & - & - & - & 0 & - & - & - & - & 1.000 & - & - & - & - \\
\hline 6 & 3189 & 2397 & 2649 & 3729 & 3982 & -52 & -135 & -108 & 4 & 31 & 0.978 & 0.963 & 0.968 & 0.988 & 0.993 \\
\hline 12 & 3145 & 2359 & 2610 & 3681 & 3932 & -96 & -186 & -157 & -35 & -6 & 0.968 & 0.946 & 0.953 & 0.983 & 0.990 \\
\hline 24 & 3080 & 2304 & 2551 & 3608 & 3855 & -161 & -276 & -239 & -84 & -48 & 0.951 & 0.917 & 0.928 & 0.974 & 0.985 \\
\hline 36 & 3041 & 2274 & 2518 & 3563 & 3807 & -200 & -346 & -300 & -101 & -55 & 0.939 & 0.895 & 0.909 & 0.969 & 0.983 \\
\hline 48 & 3024 & 2264 & 2506 & 3542 & 3784 & -217 & -398 & -341 & -94 & -36 & 0.933 & 0.881 & 0.898 & 0.969 & 0.985 \\
\hline 52.3 & 3023 & 2265 & 2507 & 3540 & 3781 & -218 & -412 & -350 & -87 & -25 & 0.933 & 0.878 & 0.896 & 0.970 & 0.987 \\
\hline 60 & 3026 & 2271 & 2512 & 3541 & 3782 & -215 & -433 & -364 & -66 & 3 & 0.933 & 0.875 & 0.893 & 0.973 & 0.991 \\
\hline 72 & 3043 & 2291 & 2531 & 3556 & 3796 & -198 & -454 & -373 & -23 & 58 & 0.938 & 0.876 & 0.896 & 0.980 & 1.000 \\
\hline 84 & 3071 & 2319 & 2559 & 3584 & 3824 & -170 & -465 & -371 & 31 & 125 & 0.949 & 0.885 & 0.905 & 0.992 & 1.012 \\
\hline 96 & 3107 & 2352 & 2593 & 3620 & 3861 & -134 & -469 & -363 & 93 & 199 & 0.965 & 0.901 & 0.921 & 1.008 & 1.028 \\
\hline
\end{tabular}

*Mean time when the newborn infant achieves the minimum weight.

Table 4 Distribution of the newborn infants according to their maximum weight change

Estimated* maximum newborn weight change (\%) n $(\%)$

$\leq-15$

$5(0.4)$

$[-15 ;-10]$

$[-10 ;-7]$

$[-7 ;-4]$

$[-4 ; 0]$

$\geq 0$

*Estimated weight change at $52.3 \mathrm{~h}$ of life.

authors concluded that definitions, types of measurements and reporting styles varied and, in most studies, daily weights measurements were not available. When they appraised the included studies, they found that the frequency of weight measurements was not comparable across studies that there were inconsistencies as to whether the day of birth was counted as day zero or day one and that the cut-off point used to define excessive weight loss was arbitrary. The authors considered that measurement bias occurred in many of the studies that met the inclusion criteria. The frequency of weighing was inadequate and the lack of measurements made determining the lowest weight and normal patterns of newborn weight change impossible. Another problem with the previous studies was that the size of the sample often varied within a study due to attrition for example a smaller sample on day three than on day one - and this fact made calculating the mean weight change more complicated (1).

Regarding the method of assessing weight change, some authors performed daily weights $(15,16)$, others only performed daily weights up to discharge $(17,18)$ or until newborn infants regained their birthweight (19). Manganaro et al. weighed the newborn infants daily until discharge and then at five and 10 days (20). DeMarzo et al. (21), Jolly et al. (22) and Macdonald et al. (23) weighed the newborn infants on the day of birth and then on specific days: days five and 10 (21); days three, seven, 10, 17 and 24 (22); and at discharge and on days five, seven, 10 and 14 (23), respectively. Only Martin-Calama et al. (24) weighed the newborn infants at more regular periods, at 6, 12, 24, 48 and $72 \mathrm{~h}$ of life, and expressed the newborn infant's age in hours. Given the diversity of the methods used, it is difficult to compare the results. Our study has the advantage of having the newborn infants weighed at different ages, ranging from 6.3 to $96 \mathrm{~h}$ of life, which allowed us to pool the data and estimate the time when the nadir was achieved.

In the aforementioned studies, newborn weight change was described as the amount and, or, timing and the diversity of descriptions made it even harder to compare study results. Weight change has been previously described as the amount in grams or kilograms lost or gained $(22,24,25)$, as the percentage of birthweight lost or gained $(15-18,20,21,23,24)$, or simply as the day when the newborn infants started to gain weight $(15,19,23)$ or when they regained their birthweight $(19,21,23)$. In addition, some authors presented the results as mean weight change $(18,20,24,25)$, median weight change $(16,23,25)$, range of weight change $(16,20,21)$, number of subjects over or under some predetermined percentage of weight loss $(17,20,21)$ or percentile data (23). Thus, we decided to describe the newborn weight change by quantity, along with reference intervals and timing. We have reported the mean weight, weight difference and weight ratio during the first $96 \mathrm{~h}$ of life, using a continuous method - a new approach - and have reported the time point when the nadir was achieved.

Regarding the timing of the nadir, Michel et al. (18) observed that it occurred between the first and second days of life, while Muskinja-Montanji et al. (15) reported that it 
happened between the second and third days of life. Both of these studies performed daily weights to the newborns, and in both, the two referred days accounted for about $90 \%$ of the sample. So, it seems that the disparity is explained by counting the day of birth as day zero or day one. If that was the case, it seems that the nadir in those studies occurred between the 24 and $72 \mathrm{~h}$ of life. To avoid these discrepancies, we think it is preferable to use the age of the newborn infants in hours instead of days. In our study, the nadir was estimated at $52.3 \mathrm{~h}$ of life, which is in accordance with the previous studies $(15,18)$. Macdonald et al. (23) performed a study with a relatively larger sample size than the previous ones $(n=937)$ and observed that the nadir occurred at a median of 2.7 days, which was also in accordance with the previous studies $(15,18)$ and with our results. Our approach has the advantage of having the newborn infants weighed with different ages, in hours, which allowed us to construct the weight change curve with reference intervals for the first $96 \mathrm{~h}$ of life and to estimate the point when the weight was minimum, which corresponds to the inflection point of the curves.

The cut-off point to define an excessive weight loss is still controversial, but some studies have defined it as a total loss $\geq 10 \%$ (1). In our sample, $6.1 \%$ of the newborn infants lost more than $10 \%$ of their birthweight, and this proportion was lower than the proportion observed in other populations. In an Italian study (20), performed in full-term breastfed newborns, this proportion was 7.7\%, and in California, among full-term singletons, it was $12 \%$ (26). In this last study (26), 5\% of the newborn infants gained weight between birth and the third day of life, which was very different from our proportion of $0.6 \%$. Differences between populations are expected, due to several aspects such as differences in birthweight, maternal age or prevalence of breastfeeding, but it can also be due to differences in study design (1). For instance, we concluded that the minimum weight is achieved at $52.3 \mathrm{~h}$ of life, while Dewey et al. (26) weighed the newborn infants on the third day of life counting the day of birth as day zero, which corresponds to 72-96 h of life, a period when newborn infants are already gaining weight.

This was the first study to evaluate the weight change in Portuguese newborn infants, and comparing with data from other populations $(20,26)$, it seems that we have a higher proportion of newborn infants in the normal range of weight. However, further research with harmonising methods is needed to compare populations.

One of the main goals of measuring newborn weight change is to establish categories of newborns that experience increased risk of health adverse outcomes. The current clinical practice guidelines assume that some weight loss is expected, but that an excessive weight loss could be a sign of inadequate milk intake. However, the cut-off point to define it varies between the recommendations: $\geq 7 \%$ loss of birthweight (27), $\geq 7 \%$ loss of birthweight or continued loss after day three or failure to regain birthweight within 10 days (28) or two to 3 weeks (29), or $\geq 8 \%$ loss of birthweight accompanied by delayed lactogenesis (30).
Considering our data, we were able to provide a weight change curve with its reference intervals corresponding to the $3 \%$ and $10 \%$ of the newborn infants with higher and lower losses. We think this can be a useful tool for clinicians, allowing them to rapidly access whether the newborn infant is experiencing excessive weight loss compared to the estimated references intervals. Also, using our statistical model, if the healthcare professional knows the birthweight and another weight of the newborn infant, for instance at 10,15 or $20 \mathrm{~h}$ of life, the weight change curve of that newborn infant for the first $96 \mathrm{~h}$ of life can be predicted, as well as the estimated minimum weight achieved, by estimating the newborn infant weight at $52.3 \mathrm{~h}$ of life. This information may allow initiating primary interventions such as a more intensive evaluation of breastfeeding technique and correcting problems, improving milk production and transfer, even before the newborn infant loses an excessive amount of weight, preventing an excessive weight loss.

\section{Strengths and limitations}

A previous systematic review (1) included studies performed among different populations, namely in Bangladesh, France, India, Italy, Jamaica, Scotland, Serbia, Spain, Sweden and the United States, but our study, besides being the first performed among Portuguese newborn infants, has a larger sample size than the previous studies. The sample sizes of those studies varied from 21 to 937 newborns, with a median of 120 .

Birthweight was recorded for clinical purposes and subsequently abstracted from the medical records, so the standard procedure could have some variations between the included maternities, but we do not expect that this fact may have impacted our results. Subsequent weight measurements were also performed prospectively and by our trained examiners.

Our methodological approach used weights performed at different time points to develop a model to estimate weight change. However, we did not have regular time point measurements for each newborn infant, instead we had one weight measurement for each newborn infant and they were weighed at different ages in hours. With this data, we developed a longitudinal model adjusted for the newborn infant's age in hours that allowed the intercept and slope to differ for each newborn infant. The fact that a systematic review (1) found a mean weight change and a nadir time point similar to ours adds support to our methodology. Most of the previous studies measured their participants at a specific time point, equal for everyone, for instance at the third day of life. However, those studies assumed that all newborn infants achieved the nadir at the same day of life; moreover, at day three, the nadir may have already occurred. Since there is a large variability in the time point when each newborn infant achieves the nadir, we believe that having newborn infants measured at different time points and then adjusting weight for the newborn infant's age in hours is a new and appropriate approach, very useful 
when adequate regular time point measurements for each newborn infant are not available, as occurred in our study.

To the best of our knowledge, this was the first study to describe the weight change during the first $96 \mathrm{~h}$ of life as a continuous variable, to provide reference intervals for this weight change and to estimate the time in hours when the newborn infants achieved their minimum weight.

\section{CONCLUSIONS}

This model allows a more accurate estimate of newborn weight change during the first days of life and its reference intervals. It estimated that the nadir occurred at $52.3 \mathrm{~h}$ of life, corresponding to a loss of $218 \mathrm{~g}$ and a weight ratio of 0.933 .

\section{ACKNOWLEDGMENTS}

The authors gratefully acknowledge the families enrolled in the Generation XXI for their kindness, all members of the research team for their enthusiasm and perseverance and the participating hospitals and their staff for their help and support.

\section{FUNDING}

Generation XXI was funded by Programa Operacional de Saúde - Saúde XXI, Quadro Comunitário de Apoio III and Administração Regional de Saúde Norte (Regional Department of Ministry of Health). It has support from the Portuguese Foundation for Science and Technology (SFRH/BD/69945/2010 and F-COMP-01-0124-FEDER011008; PTDC/SAU-ESA/105033/2008) and from the Calouste Gulbenkian Foundation. [Correction added on 9 October 2015, after online publication: Funding information for "F-COMP-01-0124-FEDER-011008" was previously incorrect and this has now been amended in this version.]

\section{References}

1. Noel-Weiss J, Courant G, Woodend AK. Physiological weight loss in the breastfed neonate: a systematic review. Open Med 2008; 2: e99-110.

2. Modi N, Betremieux P, Midgley J, Hartnoll G. Postnatal weight loss and contraction of the extracellular compartment is triggered by atrial natriuretic peptide. Early Hum Dev 2000; 59 : 201-8.

3. Singhi S, Sood V, Bhakoo ON, Ganguly NK, Kaur A. Composition of postnatal weight loss \& subsequent weight gain in preterm infants. Indian J Med Res 1995; 101: 157-62.

4. Clarke AJ, Sibert JR. Hypernatraemic dehydration and necrotizing enterocolitis. Postgrad Med J 1985; 61: 65-6.

5. Cooper WO, Atherton HD, Kahana M, Kotagal UR. Increased incidence of severe breastfeeding malnutrition and hypernatremia in a metropolitan area. Pediatrics 1995; 96 : 957-60.

6. Kaplan JA, Siegler RW, Schmunk GA. Fatal hypernatremic dehydration in exclusively breast-fed newborn infants due to maternal lactation failure. Am J Forensic Med Pathol 1998; 19: $19-22$.
7. van Dommelen P, van Wouwe JP, Breuning-Boers JM, van Buuren S, Verkerk PH. Reference chart for relative weight change to detect hypernatraemic dehydration. Arch Dis Child 2007; 92: 490-4.

8. Levene MI, Fawer CL, Lamont RF. Risk factors in the development of intraventricular haemorrhage in the preterm neonate. Arch Dis Child 1982; 57: 410-7.

9. Oh W, Poindexter BB, Perritt R, Lemons JA, Bauer CR, Ehrenkranz RA, et al. Association between fluid intake and weight loss during the first ten days of life and risk of bronchopulmonary dysplasia in extremely low birth weight infants. J Pediatr 2005; 147: 786-90.

10. Wadhawan R, Oh W, Perritt R, Laptook AR, Poole K, Wright LL, et al. Association between early postnatal weight loss and death or BPD in small and appropriate for gestational age extremely low-birth-weight infants. J Perinatol 2007; 27: 359-64.

11. Bell EF, Warburton D, Stonestreet BS, Oh W. Effect of fluid administration on the development of symptomatic patent ductus arteriosus and congestive heart failure in premature infants. N Engl J Med 1980; 302: 598-604.

12. Larsen PS, Kamper-Jorgensen M, Adamson A, Barros H, Bonde JP, Brescianini S, et al. Pregnancy and birth cohort resources in Europe: a large opportunity for aetiological child health research. Paediatr Perinat Epidemiol 2013; 27: 393-414.

13. Royston P. Calculation of unconditional and conditional reference intervals for foetal size and growth from longitudinal measurements. Stat Med 1995; 14: 1417-36.

14. Altman DG. Construction of age-related reference centiles using absolute residuals. Stat Med 1993; 12: 917-24.

15. Muskinja-Montanji G, Molnar-Sabo I, Vekonj-Fajka G. [Physiologic neonatal body weight loss in a "baby friendly hospital"]. Med Pregl 1999; 52: 237-40.

16. Marchini G, Fried G, Ostlund E, Hagenas L. Plasma leptin in infants: relations to birth weight and weight loss. Pediatrics 1998; 101: 429-32.

17. Bhat SR, Lewis P, David A, Liza SM. Dehydration and hypernatremia in breast-fed term healthy neonates. Indian J Pediatr 2006; 73: 39-41.

18. Michel MP, Gremmo-Feger G, Oger E, Sizun J. [Pilot study of early breastfeeding difficulties of term newborns: incidence and risk factors]. Arch Pediatr 2007; 14: 454-60.

19. Hossain MA, Islam MN, Shahidullah M, Akhter H. Pattern of change of weight following birth in the early neonatal period. Mymensingh Med J 2006; 15: 30-2.

20. Manganaro R, Mami C, Marrone T, Marseglia L, Gemelli M. Incidence of dehydration and hypernatremia in exclusively breast-fed infants. J Pediatr 2001; 139: 673-5.

21. DeMarzo S, Seacat J, Neifert M. Initial weight loss and return to birth weight criteria for breast-fed infants: challenging the "rule of thumb" [conference proceeding]. Am J Dis Child 1991; 17: 31-8.

22. Jolly PE, Humphrey M, Irons BY, Campbell-Forrester S, Weiss HL. Breast-feeding and weight change in newborns in Jamaica. Child Care Health Dev 2000; 26: 17-27.

23. Macdonald PD, Ross SR, Grant L, Young D. Neonatal weight loss in breast and formula fed infants. Arch Dis Child Fetal Neonatal Ed 2003; 88: F472-6.

24. Martin-Calama J, Bunuel J, Valero MT, Labay M, Lasarte JJ, Valle $\mathrm{F}$, et al. The effect of feeding glucose water to breastfeeding newborns on weight, body temperature, blood glucose, and breastfeeding duration. J Hum Lact 1997; 13: 209-13.

25. Rodriguez G, Ventura P, Samper MP, Moreno L, Sarria A, Perez-Gonzalez JM. Changes in body composition during the 
initial hours of life in breast-fed healthy term newborns. Biol Neonate 2000; 77: 12-6.

26. Dewey KG, Nommsen-Rivers LA, Heinig MJ, Cohen RJ. Risk factors for suboptimal infant breastfeeding behavior, delayed onset of lactation, and excess neonatal weight loss. Pediatrics 2003; 112: 607-19.

27. American Academy of Pediatrics. Breastfeeding and the use of human milk policy statement. Pediatrics 2005; 115: 496-506.
28. International Lactation Consultant Association. Clinical guidelines for the establishment of exclusive breastfeeding. Raleigh (NC): ILCA, 2005.

29. Registered Nurses Association of Ontario. Breastfeeding best practice guidelines for nurses. Toronto (ON): RNAO, 2003.

30. The Academy of Breastfeeding Medicine. ABM Protocol 3: Hospital guidelines for the use of supplementary feedings in the healthy term breastfed infant. 2007. 